\title{
Twitter User Behaviors In Turkey: A Content Analysis On Turkish Twitter Users
}

\author{
Burak Polat \\ Marmara University Faculty of Communication \\ aloha@burakpolat.com \\ Cemile Tokgöz \\ Marmara University Faculty of Communication \\ cemiletokgoz@gmail.com
}

\section{DOI:10.5901/mjss.2014.v5n22p244}

\begin{abstract}
Social media, as an interactive and digital communication medium that is relatively free of time and place boundaries, is transforming the way we communicate. Since the Internet fully commercialized by 1995, web based technologies those constitutes social media have been developed with an accelerated pace. Relatively proportional to technology development pace, adoption rates of social media tools such as web logs (blogs), online social networks, social bookmarking sites, etc. are extremely high and Twitter, an online social networking and micro-blogging service, is a significant example with its hundred millions of users. Since the most of the Twitter penetration rates are from Turkey, obviously Twitter has been a popular social media channel in Turkey. In this article; at first interaction, the fundamental concept of social media, will be defined in depth and then a content analysis on Turkish dataset from Twitter will be shared to identify the user behaviors in Turkey.
\end{abstract}

Keywords: Social Media, User Behviours, Twitter Content Analysis, Behavioural Research, Interactivity

\section{Introduction}

Marshall Mcluhan's famous quote "Medium is the message.", with the influence of Social Media, is transformed into "User is the message.". We are living in a age that individual's influence on media is rising every passing day. Social Media, as a multi-directional new communication medium that is relatively free of time and place boundaries, enables users to create, share and interact with the digital content thus each user is a part of the audience and also broadcaster at the very same time.

The dynamics of the social media allows user-generated content to be amplified and spread to the mass. The flow of content diffusion starts with creating and sharing the content, however interactivity makes social media literally social thus content epidemically diffuses with interactions.

Twitter is one of the major social media tools that enables to create, share and interact with digital content. The structure of the online social network gives users a potential to be heared. In this research, it is aimed to understand user behaviours on creating, sharing and interacting with the content. According to this aim, at first social media as a new media, interactivity concepts and also Twitter as a social media tool will be defined in depth. Then a content analysis on \#TwitterforlCSS hashtag and participants' Twitter usage data will be analyzed and shared to actualize the aim.

\section{Social Media, Interactivity and Twitter}

New media is a term that was suggested in researches about social, pshpsychological, economical, political and cultural studies by 1970s. But in 1990s, the term of new media became clear with the developments on computer and internet technologies. Because new media cannot be thought without digitalization. New media has the ability to collect all of multimedia such as text, image, video, audio and, transforms audience to users. Because users can create, share and transmit content. New media provide to users interactivity. Interactivity create individuality. Because every user transforms a source, content creater.

Lev Manovich formulates new media via five principles: (Manovich, 2002) 
Numerical Presentation: A new media object can be described mathematically. For instance, an image or a shape can be described using a mathematical fuction.

Modularity: New media sub-elements such as images, sounds, movies are represented as collections of discrete samples.

Automation: New media objects can automate many things without user.

Variability: New media allows us to create versions of the same object that differ from each other in substantial ways.

Transcoding: Computer data is represented in variable formats. Mostly formats are recognized by each other.

The most pervasive type of new media is social media. Social media is formed user generated contents and interactivity.

The global system of networked computers, servers and routers known as the Internet has transformed many aspects of modern society and social interaction. The online distribution of goods and services, for instance, has influenced almost every industry and has radically transformed many. Alongside commerce-oriented technological development has been a rise in what has been termed "social media." One of the most significant developments connected to social media is the rise of social network sites, such as Facebook, Linkedln, Twitter and Google Plus. (Boyd\&Ellison, 2013)

Boyd and Ellison assert that Social Networks have three essential components:

1) a user-constructed public or semi-public profile;

2) a set of connections to other users within the system;

3) the ability to view one's own list of connections, as well the connections made by others in the system. (Boyd\&Ellison, 2007)

In new media, interactivity creates a control field to users and behaviours of users became quality of source. Thus, communication became user-oriented. Interactivity is the key element for understanding user-generated social media.

\section{Interactiviy}

Interactivity is two or more way communication. Messages between source and receiver forms some sort of influence. This influence causes the receiver to react and give feedback. The feedback forms another message. Thus receiver becomes the source and sourcebecomes the receiver. It goes on until the end of communication. (Öğüt, 2004)

Interactivity is a process-related, variable characteristic of communication settings. Interactivity can lead to sociability. Interactivity is not a characteristic of the medium. It is a process-related construct about communication. we note that communication is mostly about and for the purpose of interaction. Interactivity places shared interpretive contexts in the primary role. Interactivity describes and prescribes the manner in which conversational interaction as an iterative process leads to jointly produced meaning. Interactivity is the condition of communication in which simultaneous and continuous exchanges ocur, and these exchanges carry a social, binding force. (Rafaeli \& Sudweeks, 1997)

The aim for interactivity in new media is obviously the need of user involvement. This is an extension of the need for reaching information quick and accurately. (Öğüt, 2004)

According to Mathematical Theory of Communication, information source produces a message; transmitter that operates on the message to create a signal which can be sent through a channel; channel carries the information that composes the message; receiver tranforms signal back into the message intended for delivery; and finally destination takes the message. If there is a noise in channel, message cannot be transmit accurately (Shannon, 1948) This mathematical approach disregards content of the message. In the aspects of social siences, content of the message is significant and message should has the effectiveness. According to Umberto Eco, if receiver could not understand the content of the message, there is a kind of noise.

The aim of communication is not only transmit a message to receiver, and also create content. Thus, interactivity is the most significant feature of new media, because it makes the content effective.

The definition of interactivity according to Rafaeli recognizes three pertinent levels: (Rafaeli, 1988)

Two-way Communication (noninteractive) is present as soon as messages flow bilaterally.

Reactive Communication settings require later messages refer to earlier ones. 
Fully Interactive Communication differs from reaction in the incorporation of reference to the content, nature, form or just the presence of earlier reference.

Not all communication is interactive and even noninteractive communication may contain coherent responses. Interactivity is not a medium characteristic. Media and channels may set upper bounds, remove barriers, or provide necessary conditions for interactivitiy levels. But potential does not compel actuality.

Interactivity is feedback that relates both to previous messages and to the way previous messages related to those preceding them.

Wiberg states that the concept of interaction and interaction support could be defined in relation to the concepts of communication and collaboration. Wiberg clarifies his model on defining the terms, communication as the exchange of information between people via a tool / medium and collaboration as two or more people operating a common object or artifact. In collaboration, operations produce "feedback" to the operator, but also "feed through" to co-workers. He states that in the context of his suggested model, communication and collaboration could be concived as subsets of interaction. (Wilberg, 2004) (Öğüt, 2011)

\section{From Passive Audience to Active User By New Media:}

With new media, audience of mass media transforms user. The users is the key element in new media. Because, receiver becomes source again, and users create content in new media. Content creation provides the user perception of freedom and selection oppotunity.

Differences between active and passive users will be given over Biocca's audience activity approach (Biocca, 1988). Because, differences between active and passive users also show the differences between new media and mass media users.

Freedom of selection: User activity is portrayed as the funneling process of media and content selection.

Utilitarianism: The audience member is the embodiment of the self-interested consumer. Beyond selectivity, which in some cases implies a certain defensiveness on the part of the user, the utilitarian version of the concept suggest a certain level of rational choice in the satisfaction of clear individual needs and motives.

Intentionality: It is in this form that the concept emphasizes the more cognitive dimensions of activity. Intentionality points to schematic processing and structuring of incoming information. Patterns of media consumption and memory bear the clear imprint of the users motivation, personality and individual cognitive processing structure.

Involvement: Involvement characterize both the level of affective arousal and a level of cognitive organization and information structuring. The same activity term is further used to label behavioral manifestations of active involvement such as parasocial interaction.

Imperviousness to Influence: It functions as a kind of goal of activity by reference to the degree to which the user limits, influences and controls the effects media.

Twitter

Twitter is an online social networking and microblogging service that enables users to send and read short 140-character text messages, called "tweets". The first Twitter prototype, developed by Dorsey and contractor Florian Weber, was used as an internal service for Odeo employees and the full version was introduced publicly on July 15, 2006.http://en.wikipedia.org/wiki/Twitter - cite_note-launch-9Twitter describes itself as a best way to connect with people express yourself and discover what's happening. Twitter has become a news reporting, coordination and social media marketing tool. Features of Twitter:

Tweets: Tweets are publicly visible by default, but senders can restrict message delivery to just their followers. (Wikipedia, 2014):

Retweet (RT): A retweet is someone else's Tweet that you chose to share with all of your followers. (Twitter, 2014)

Favourite: Users use this feature for tweets that they like. (Twitter, 2014)

Format: Users can group posts together by topic or type by use of hashtags - words or phrases prefixed with a \# sign. Similarly, the @ sign followed by a username is used for mentioning or replying to other users. (Wikipedia, 2014) 
Trending Topics: A word, phrase or topic that is tagged at a greater rate than other tags is said to be a "trending topic". Trending topics become popular either through a concerted effort by users, or because of an event that prompts people to talk about a specific topic. These topics help Twitter and their users to understand what is happening in the world. (Wikipedia, 2014)

Direct Messages (DM): Users can directly send or take messages from followers. These messages are not be seen on the timeline. (Twitter, 2014)

The most commonly used social networking platform of the five measured by Duggan and Smith. Facebook also high levels of engagement among its users: $63 \%$ of Facebook users visit the site at least once a day, with $40 \%$ doing so multiple times throughout the day. Instagram and Twitter have a significantly smaller number of users than Facebook does, but users of these sites also tend to visit them frequently. Some $57 \%$ of Instagram users visit the site at least one a day (with $35 \%$ doing so multiple times per day), and $46 \%$ of Twitter users are daily visitors (with $29 \%$ visiting multiple times per day). (Duggan\&Smith, 2013)

Overall, $42 \%$ of online adults use two or more of these social networks, while $36 \%$ use only one. Among those who only use one major social networking platform, $84 \%$ say that Facebook is the single site that frequent. However, other single platform social networking site users have adopted a site other than Facebook as their platform of choice. Among those who use just one social networking site, $8 \%$ Ise Linkedln, $4 \%$ use Pinterest, and $2 \%$ each say that Instagram or Twitter is their social networking site.

\section{Content Analysis on Turkish Twitter Users Methodology}

For determining Twitter user behaviours in Turkey an experiment is designed; a hashtag, \#TwitterforlCSS, is created and tweeted by the authors to ask users to participate with the research with a straightforward question such as "Why do you use Twitter?". Users has been told that the participation will allow authors to collect users' data from Twitter. In a week, 63 users participated with 76 tweets; \#TwitterforlCSS hashtag's and participants' twitter usage data is gathered from 22.06.2014 to 28.06.2014, the data collection categories can be listed as:

\section{a. \#TwitterforICSS}

a.1. Tweets

a.1.1 Tweets' Time and Date

a.1.2 Interactions (Quantity of Retweets, Favorites and Mentions for each Tweet)

b. User Twitter Usage

b.1. Tweets

b.1.1. Tweets' Time and Date

b.1.2. Interactions

b.2. Users' Information

b.2.1. Quantities of Retweets and Favorites

b.2.2. Quantity of Total Tweets, Photo/Video Sharing, Followings/Followers

b.2.3. Twitter Registiration Date

b.2.4. Location

b.2.5. Gender and Age (Gathered from Users' themself)

From dataset a, participants' Twitter perception will be identified by concentrating responses. From dataset $b$, interaction behaviours will be identified according to topics, distrubuted multimedia elements, gender, age and time.

\section{Participants}


63 Twitter users participated with the \#TwitterforlCSS hashtag. The demographies of the participants is shown at table 1.
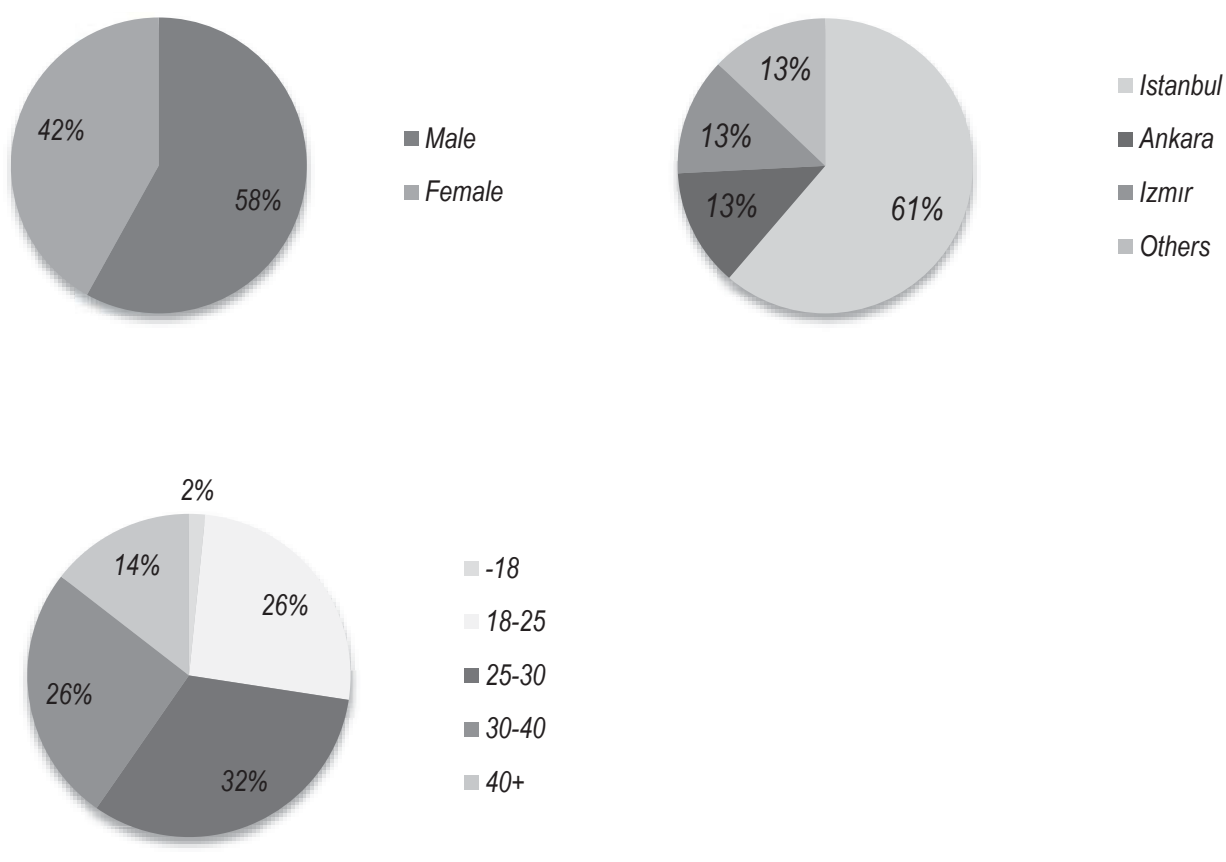

Table 1: Gender, Location and Age Distribution of Participants

\section{Results}

a. Content Analysis on \#TwitterforICSS

Participants responded to the "Why do you use Twitter?" question with their tweets, after concentrating the responses 14 headings became distinct;

\begin{tabular}{|l|l|l|}
\hline Self-expression tool & 17 & $13 \%$ \\
\hline Free media & 11 & $8,45 \%$ \\
\hline Fast & 22 & $17 \%$ \\
\hline Only one of the social networks & 4 & $3 \%$ \\
\hline Filtering by interests & 8 & $6.15 \%$ \\
\hline Source of information/news & 22 & $17 \%$ \\
\hline Not an efficient online social network & 1 & $0,75 \%$ \\
\hline Public Sphere & 9 & $7 \%$ \\
\hline Entertainment/Humor & 5 & $3.85 \%$ \\
\hline Actuality & 14 & $10.75 \%$ \\
\hline Discussion/Learning & 6 & $4.7 \%$ \\
\hline Opportunity to reach opinion leader/celebrities & 3 & $2.3 \%$ \\
\hline
\end{tabular}




\begin{tabular}{|l|l|l|}
\hline Reliable & 3 & $2.3 \%$ \\
\hline Simple/easy to read & 5 & $3.85 \%$ \\
\hline
\end{tabular}

Table 2 - "Why do you use Twitter?" Responses at \#TwitterforlCSS

According to this research, most of participants expressed that Twitter is a fast social network and is a source of information/news. For each of two features has $17 \%$ participation. Twitter as a self-expression tool is seen by $13 \%$ of participants. Actuality of Twitter follows these features with $10.75 \%$ and, $8.45 \%$ of participants think that Twitter is a free medium. $7 \%$ of participants comment Twitter as a public space. From this point of view, Twitter is perceived as a fast source of news and free public space.

$6.15 \%$ of participants emphasized the filtering feature of Twitter. Because Twitter provide the opportunity of filtering for interests by hashtags. In addition, $4.7 \%$ of participants see Twitter as a learning platform by discussion with the others. Thus, Twitter is a medium that provide communication with people who has the same interest and learning something with discussing about these interest fields.

$3.85 \%$ of participants emphasize Twitter as an entertainment or humor medium. Especially sharing photos and videos provides this feature of Twitter. And also $3.85 \%$ of participants expressed that Twitter is a simple and easy to read. It makes this medium easy to follow. $3 \%$ of participants think that Twitter is an only one of the social networks. According to $2.3 \%$ of participants, they reach the opportunity to communicate with opinion leaders and celebrities. And $2.3 \%$ of them point out that Twitter is a reliable platform. Finally only $0.75 \%$ of participant see Twitter loses its function as an effective social network.

b. Twitter User Behaviours

63 participants' Twitter data from 22.06.2014 to 28.06.2014, (Users' information and 588 unique tweets with their interactivity metrics) are gathered. Significant results are;

Total tweets tweeted by the user and follower quantity of the user is weakly correlated ( $R=0.222, R 2=0.0493)$; thus more tweeting does not necessarily mean more followers.

$\% 58$ of the tweets have humorous content. According to category distribution of humorous contents, users are tend to make humorous comments on politics.

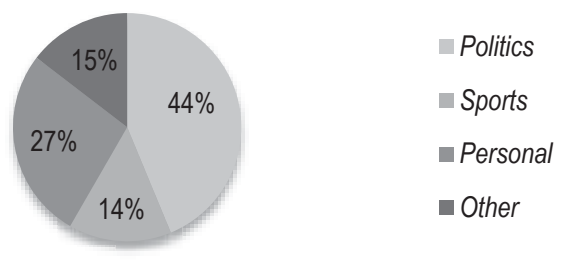

Table 3 - Category Distrubiton of Humorous Content

Twitter Interactivity is provided by actions such as Favorite, Retweet and Mention; the overall interactions of 588 tweets is shown at table 3.

Table 4 - Interactive of Tweets Gathered

\begin{tabular}{|l|l|l|}
\cline { 2 - 3 } \multicolumn{1}{c|}{} & Total & Average \\
\hline Favorite & 2718 & 4,62244898 \\
\hline Retweet & 2482 & 4,221088435 \\
\hline Mention & 613 & 1,042517007 \\
\hline
\end{tabular}


The quantity of interaction activities in each tweet (retweets, favorites and mention) is summed up with equal weight to generate an interaction score. Results according to the Interaction score;

Distributed multimedia elements effect on tweets' interactivity:

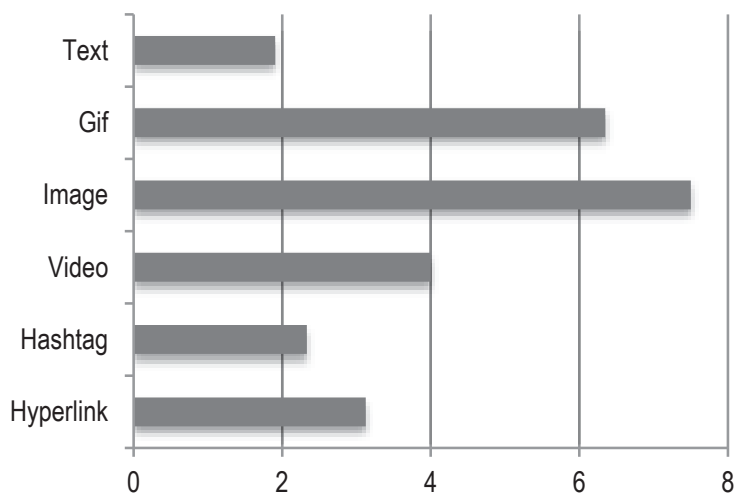

Table 5 - Multimedia Elements Effect on Interactivity [Multimedia Elements (y), Average Interaction Score (x)] Humorous contents' effect on interactivity:

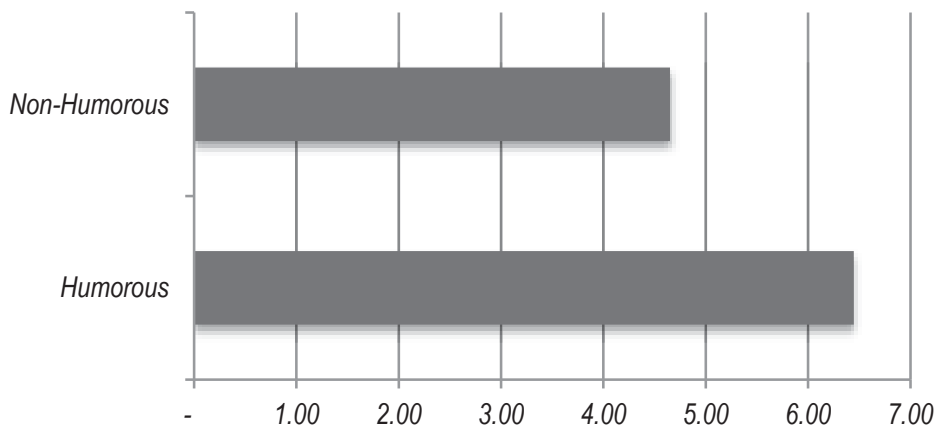

Table 6 - Humorous contents' effect on Interactivity [Content Type (y), Average Interaction Score (x)]

\section{Research Limitations}

The quantity of participants and gathered data within the corresponding time interval can not be considered as a sample for Turkish Twitter Users universe, in this manner the results will only enlighten a path for further researches with larger datasets. In further researches a digital tool, that enables gathering larger datasets from Twitter, will be used to provide a sample to represent the related universe.

\section{Discussions and Further Research Questions}

Social Media, as a New Media, is an interactive communication medium that enables users to communicate digitally relatively free of time and place boundaries. With the power of social media tools such as Twitter, individuals' voices is getting heard more every passing day as considerable amount of participants emphasize. Interactivity in social media is an amplifier that amplifies the opinions so understanding the dynamics of interactivity will allow individuals to reach the mass.

According to this point of view, our research is shaped. From the considerable amount of participation in \#TwitterforICSS hashtag, Twitter is considered as a free medium that enables users to express themselves effectively. Twitter usage data of the participants gathered significantly showed us more tweets does not necessarily means more influence. Humorous 
contents interactivity effect is emphasized and in researched universe strong relationship between humor and politics contents is came forward. According to distributed multimedia elements effect is shared and content that contains images' significant interaction potential is observed.

Because of the time and resource limitations, our research covered only a small universe and results shared are not responding our aim of understanding the dynamics of interactivty. However this research enlightens a path for further researches; with a sufficient dataset and time interval, quality of tweets' effect on interactivity, motivations of user interactivity, typology of users according to their tweets and interactions and interactivity according to content types will be covered in depth.

\section{References}

Biocca, F.A., (1988) Opposing Conceptions of the Audience: The Active and Passive Hemispgeres of Mass Communication Theory, Anderson, J.A. (Ed.), Communication Yearbook 11, p.51-80, US: Sage Publications.

Boyd, D. \& Ellison, N.B., (2007) Social Network Sites: Definition, History and Scholarship, Journal of Computer-Mediated Communication, Vol.13, Issue 1, p210-230, Blackwell.

Boyd, D. \& Ellison, N.B., (2013) Sociality Through Social Network Sites, Dutton, W.T. (Ed.), The Oxford Handbook of Internet Studies, Oxford University Press, 2013.

Duggan, M. \& Smith, A., (2013) Social Media Update 2013.

Manovich, L., (2002) The Language of New Media, Leonardo Books.

Öğüt, S., (2004) Interactivity in New Media, MA Thesis, Yeditepe University, İstanbul.

Öğüt, S., (2011) Control On Users: A Critical Approach on Interaction, New Media Conference, Yeditepe University.

Rafaeli, S., (1988) Interactivity: From New Media to Communication, Sage Annual Review of Communication Research: Advancing Communication Science, Vol.16.

Rafaeli, S. \& Sudweeks, F., (1997) Networked Interactivity, Journal of Computer-Mediated Communication, Vol.2, Issue 4. Shannon, C.E., (1948) A Mathematical Theory of Communication, The Bell System Technical Journal, Vol.27, p.379-423.

Twitter. (2014, June 27). Wikipedia. Retrieved June 29, 2014, from http://en.wikipedia.org/wiki/Twitter

Types of Tweets and where they appear. (n.d.). Twitter Help Center. Retrieved June 29, 2014, from

https://support.twitter.com/articles/20169592-tweet-turleri-ve-gorundukleri-yerler\#

Wiberg, M., (2004) Introduction-The Emerging Interaction Society, Interaction Society: Theories, Practice and Supportive Technologies, Information Science Publishing, p.1. 